\title{
Quantitative Determination of Renal Vascular Changes Related to Age and Hypertension
}

\author{
Takenori Yamaguchi, M.D., Teruo Omae, M.D., and \\ Shibanosuke KATsuki, M.D.
}

\begin{abstract}
SUMMARY
In 101 autopsy cases, the degrees of stenosis, hyalinization, and fibroelastic hyperplasia of arterioles and small arteries of the kidney were quantitatively determined and the correlations of each change with age and with antemortem blood pressure level were studied. Renal vascular changes are caused by factor(s) related to age, but markedly accelerated by hypertension. In the presence of hypertension the progression of stenosis of arterioles is nearly the same as that of small arteries. Hyalinization of arterioles and fibroelastic hyperplasia of arterioles and of small arteries progress with age, but hyalinization of arterioles is related more closely to hypertension than to aging, and fibroelastic hyperplasia is more closely related to aging than to hypertension.

The degree of stenosis of renal vessels is more closely correlated with systolic than with diastolic blood pressure, particularly in small arteries; arteriolar hyalinization is somewhat more closely correlated with an increase in diastolic than in systolic blood pressure. The correlation between fibroelastic hyperplasia and blood pressure is relatively poor.
\end{abstract}

\section{Additional Indexing Words :}

Nephrosclerosis Cardiac hypertrophy Renal arteriole

\begin{abstract}
LTHOUGH it has been well established that there is a high incidence A of renal arteriolar sclerosis in patients with hypertension, the significance of renal vascular changes for the maintenance or causation of hypertension is not clear. Discrepancies among the findings reported in the literature are due to differences both in the material studied and the methods used to assess renal arteriolar changes. The present study was designed to make quantitative determinations of the degenerative changes in arterioles and small arteries of the kidney, and to examine the specificity of each change in relation to hypertension. Degenerative changes were quantitated as to degrees of stenosis, hyalinization, and fibroclastic hyperplasia.
\end{abstract}

From the Second Department of Internal Medicine (Director: Prof. Dr. S. Katsuki), Faculty of Medicine, Kyushu University, Fukuoka.

The study was supported in part by a research grant from the Japanese Ministry of Education.

Received for publication February 10, 1969. 


\section{Materials AND Method}

\section{Materials :}

From 1,965 cases autopsied in the Department of Pathology, Kyushu University Hospital between 1961 and 1965, 101 cases were selected in which ante-mortem blood pressure levels had been recorded serially. Excluded were cases having had diseases known to cause hypertension, such as primary renal disease, hyperthyroidism, or other endocrine disorders. Because cardiac hypertrophy was used as a measure of pre-existing hypertension, those cases with valvular heart disease, severe anemia, and other conditions that affect heart weight were also excluded. The 101 cases included 55 males and 46 females, with ages ranging from 21 to 92 years (average age 59). The most frequent cause of death was found to be cerebrovascular disease, in 40 cases.

\section{Classification of Blood Pressure Levels :}

Ante-mortem blood pressure levels were classified as follows:

Group I (normotension): Blood pressure was always within normal limits, with systolic blood pressure below $140 \mathrm{~mm} . \mathrm{Hg}$ and diastolic below $90 \mathrm{~mm} . \mathrm{Hg}$, as proposed by WHO." This group included 27 cases (average age 53 years).

Group II (intermittent hypertension): Systolic and/or diastolic blood pressure exceeded at times the upper limits of normal. This group included 29 cases (average age 59 years).

Group III (persistent hypertension): Systolic and/or diastolic blood pressure was always recorded above the upper limits of normal. This group included 45 cases (average age 63 years).

Blood pressure measurements recorded within one month of death were not considered for the classification. An average of all the determinations of systolic and diastolic blood pressure was obtained to correlate with renal vascular changes in each case.

\section{Histopathological Evaluation of Renal Vascular Changes:}

Tissue blocks more than $2 \mathrm{~cm} .^{2}$ were obtained from horizontal sections of the kidney and fixed with Zenker-formol. Serial sections $7 \mu$. thick were stained with hematoxylin-eosin, Elastica-Van Gieson, PAS, and Mallory's azan methods. Arteries with a diameter less than $60 \mu$. were considered arterioles, and those between 61 and $200 \mu$. were considered small arteries. Twenty to 30 arterioles and a similar number of small arteries were examined in each specimen. A quantitative assessment of degenerative changes was made as follows (Table I) :

(1) Degree of Stenosis: The external diameter (not including adventitia) and the internal diameter were measured for each vessel, sectioned at nearly right angles to the longitudinal axis. Two measurements of each vessel were made at the largest and smallest diameters, and averaged. An assessment of the degree of stenosis was obtained by dividing the inside diameter (L) by the wall thickness (W), as described by Kernohan et al. ${ }^{2)}$ The L/W ratios of all the arterioles and small arteries in a specimen were averaged.

(2) Hyalinization: Both fibro-necrotic hyalinization and angionecrosis as described by $\mathrm{Mukai}^{3)}$, were considered as hyalinization. Hyalinization appeared 
Table 1. Quantitative Assessment of the Changes in Arterioles and Small Arteries of the Kidney

1) Degree of stenosis

$=$ Internal diameter (L)/wall thickness (W)

Wall thickness $=$ (external diameter-internal diameter $) / 2$

Note: Internal and external diameters (excluding the adventitia) were obtained by averaging the largest and smallest cross-sectional diameters of each vessel.

2) Hyalinization

0 : Normal.

1+: Hyalinization involving only a part of the subintima.

$2+$ : Hyalinization involving almost the entire circumference of the subintima or extending to the media.

$3+$ : Hyalinization involving almost the entire vessel wall.

3) Fibroelastic hyperplasia

0 : No change in the internal elastic membrane and no increase in connective tissue in the vessel wall.

1+: Internal elastic membrane thickened and disrupted into two layers or fragmented, or slight increase in connective tissue in the vessel wall.

$2+$ : Internal elastic membrane disrupted into more than three layers, among which collagen fibers are increased.

Thickness of intima does not exceed that of media.

$3+$ : Change in internal elastic membrane is more severe than $2+$ and thickness of intima exceeds that of media.

as homogeneous and structureless material, stained red by hematoxylin-eosin, yellow or yellowish-brown by Van Gieson's stain, and either blue or red by Mallory's azan stain. Each vessel was coded from 0 to 3 according to the criteria shown in Table I. The coded values for each specimen were added and divided by the number of vessels examined to obtain a measure of the degree of hyalinization.

(3) Fibroelastic Hyperplasia: Fibroelastic hyperplasia corresponds to the so-called "arteriosclerotic change" and consists of disruption, fragmentation or proliferation of the lamina elastica interna, accompanied by an increase in collagen fibers and other elements of connective tissue. The grading of this change was similar to that of hyalinization (Table I).

IV. Grading of Cardiac Hypertrophy (Cardiac index):

A measure of cardiac hypertrophy, as an index of antemortem hypertension, was obtained by dividing the heart weight in grams by the body length in centimeters, determined at the time of autopsy. ${ }^{4}$

\section{Results}

\section{Control Values}

The values of the cardiac index and renal vascular changes determined for 17 normotensive subjects below 60 years of age were used as controls. The cardiac index in control subjects was $1.62 \pm 0.27$ (mean \pm S. D.). The degree 
Table II. Renal Vascular Changes in Normotensive Subjects by Age

\begin{tabular}{|c|c|c|c|c|c|c|c|c|}
\hline \multirow{2}{*}{ Age } & \multirow{2}{*}{$\begin{array}{l}\text { No. of } \\
\text { cases }\end{array}$} & \multirow{2}{*}{$\begin{array}{l}\text { Cardiac } \\
\text { index }\end{array}$} & \multicolumn{2}{|c|}{ Degree of stenosis } & \multicolumn{2}{|c|}{ Hyalinization } & \multicolumn{2}{|c|}{$\begin{array}{l}\text { Fibroelastic } \\
\text { hyperplasia }\end{array}$} \\
\hline & & & Arterioles & $\begin{array}{l}\text { Small } \\
\text { arteries }\end{array}$ & Arterioles & $\begin{array}{l}\text { Small } \\
\text { arteries }\end{array}$ & Arterioles & $\begin{array}{c}\text { Small } \\
\text { arteries }\end{array}$ \\
\hline$<60$ & 17 & $\begin{array}{l}1.62 \\
\quad \pm 0.27\end{array}$ & $\begin{array}{l}2.52 \\
\quad \pm 0.21\end{array}$ & $\begin{array}{l}3.91 \\
\quad \pm 0.59\end{array}$ & $\begin{array}{l}0.06^{*} \\
\quad \pm 0.09\end{array}$ & $\begin{array}{l}0.01 \\
\quad \pm 0.03\end{array}$ & $\begin{array}{l}0.06 \\
\quad \pm 0.12\end{array}$ & $\begin{array}{l}0.23 * * \\
\quad \pm 0.16\end{array}$ \\
\hline$\geqq 60$ & 10 & $\begin{array}{l}1.73 \\
\quad \pm 0.31\end{array}$ & $\begin{array}{l}2.66 \\
\quad \pm 0.59\end{array}$ & $\begin{array}{l}3.84 \\
\quad \pm 1.25\end{array}$ & $\begin{array}{l}0.30^{*} \\
\pm 0.31\end{array}$ & $\begin{array}{l}0.03 \\
\quad \pm 0.08\end{array}$ & $\begin{array}{l}0.21 \\
\quad \pm 0.20\end{array}$ & $\begin{array}{l}1.00^{* * *} \\
\pm 0.56\end{array}$ \\
\hline
\end{tabular}

of stenosis was $2.52 \pm 0.21$ for arterioles and $3.91 \pm 0.59$ for small arteries. The degree of hyalinization was $0.06 \pm 0.09$ and $0.01 \pm 0.03$ and of fibroelastic hyperplasia $0.06 \pm 0.12$ and $0.23 \pm 0.16$ for arterioles and small arteries, respectively. If the value exceeding the mean $\pm 2 \mathrm{~S}$. $\mathrm{D}$. is considered abnormal, the upper limit of normal for the cardiac index was 2.16, for hyalinization 0.24 and 0.07 , and for fibroelastic hyperplasia 0.30 and 0.55 , for arterioles and small arteries respectively. In regard to the grading of stenosis, a value below 2.10 for arterioles or 2.73 for small arteries was considered abnormal; as the figure becomes smaller, the stenosis is more severe.

In 10 normotensive subjects aged 60 years or more, the cardiac indices and the degrees of stenosis in arterioles and small arteries did not differ substantially from those of the subjects less than 60 years of age, but hyalinization and fibroelastic hyperplasia were more advanced in the former than in the latter. The differences between the degrees of hyalinization in arterioles and of fibroelastic hyperplasia in small arteries in the two age groups were statistically significant (Table II).

\section{Renal Vascular Changes in Hypertension}

The relations between renal vascular changes, blood pressure and age are shown in Fig. 1.

(1) Degree of Stenosis: The degree of stenosis of arterioles and small arteries worsened from Group I to Group III. For arterioles, the degrees of stenosis for Groups I, II, and III averaged 2.52 $\pm 0.21,2.10 \pm 0.44$, and $1.51 \pm 0.62$ respectively in subjects less than 60 years of age and $2.66 \pm 0.59$, $2.32 \pm 0.57$, and $1.97 \pm 0.56$ in those over 60 . Stenosis was always greater in the younger subjects than in the older. In Group III, the difference between the two age groups was statistically significant $(p<0.05)$. For small arteries, the degrees of stenosis for Groups I, II, and III averaged 3.91 $\pm 0.59,3.36 \pm$ 0.90 , and $2.26 \pm 1.08$ respectively in subjects less than 60 years of age, and 


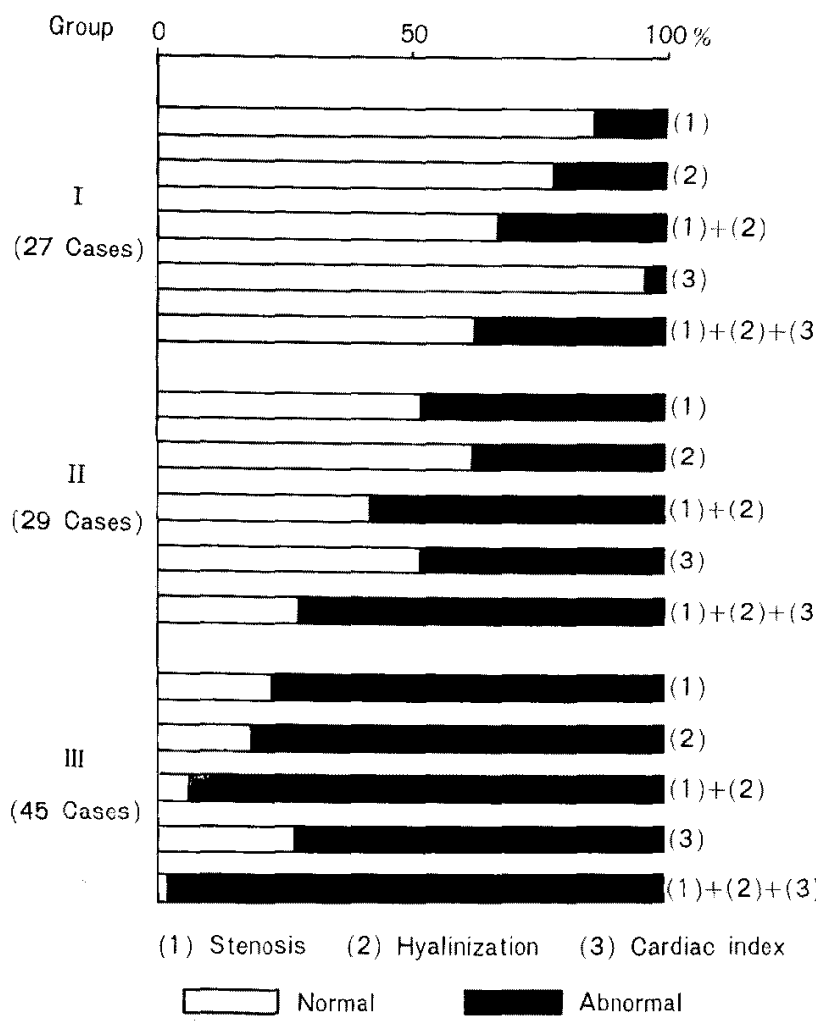

Fig. 1. Renal vascular changes and cardiac index related to age and blood pressure.

$3.84 \pm 1.25,3.13 \pm 0.92$, and $2.52 \pm 0.93$ in those over 60 .

(2) Hyalinization: The degrees of hyalinization of both arterioles and small arteries also advanced from Group I to III, and was more marked in arterioles than in small arteries. For arterioles, the degrees of hyalinization for Groups I, II, and III were $0.06 \pm 0.09,0.30 \pm 0.52$, and $1.24 \pm 0.68$ respectively in subjects less than 60 years of age and $0.03 \pm 0.31,0.34 \pm 0.44$, and $0.79 \pm 0.65$ in those over 60 . In Group III arteriolar hyalinization was more severe in the younger subjects than in the older $(p<0.05)$. Presumably, this was caused by more advanced hypertensive disease in the younger subjects, as indicated by the average blood pressure level of $174 / 106 \mathrm{~mm} . \mathrm{Hg}$ for the younger subjects, compared with that of $180 / 98 \mathrm{~mm} . \mathrm{Hg}$ for the older ones. Hyalinization was more marked in the older subjects of Group I, but in Group II, hyalinization was nearly the same for both age groups, and not substantially different from that of the older subjects of Group I. These findings suggest that although hyalinization occurs to some extent as an aging process it is markedly accelerated by hypertension. For small arteries the degrees of 
Degree of stenosis

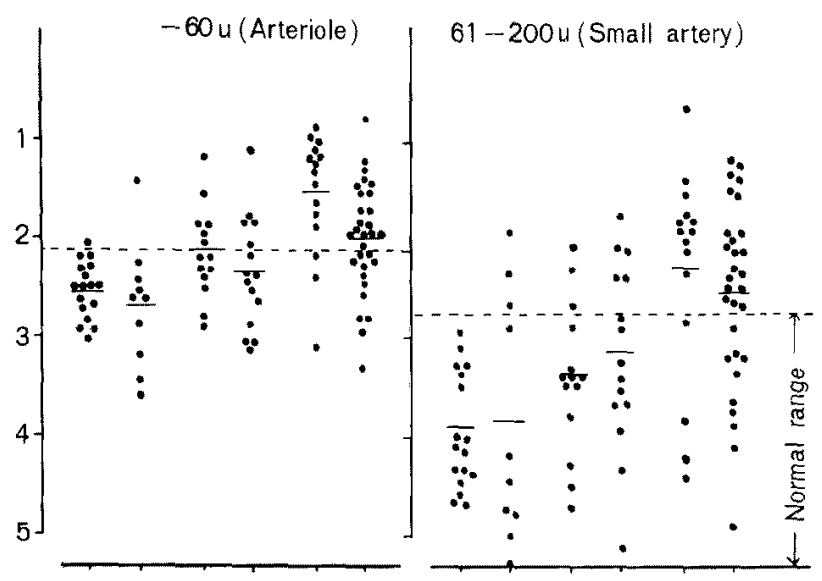

Hyalinization

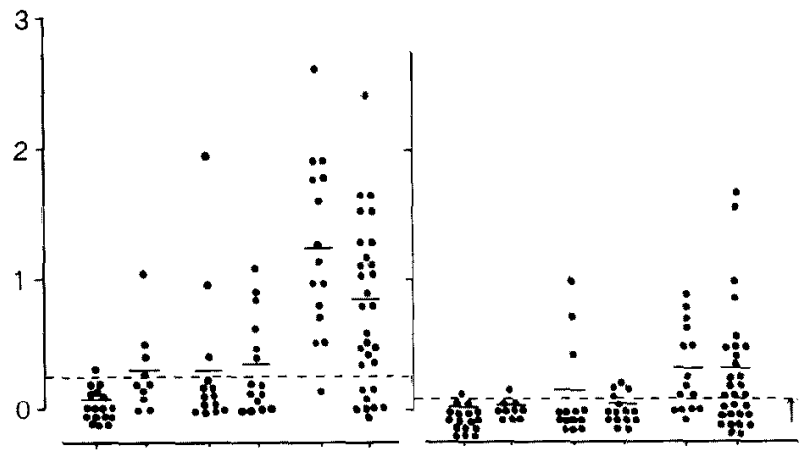

Fibroelastic hyperplasia

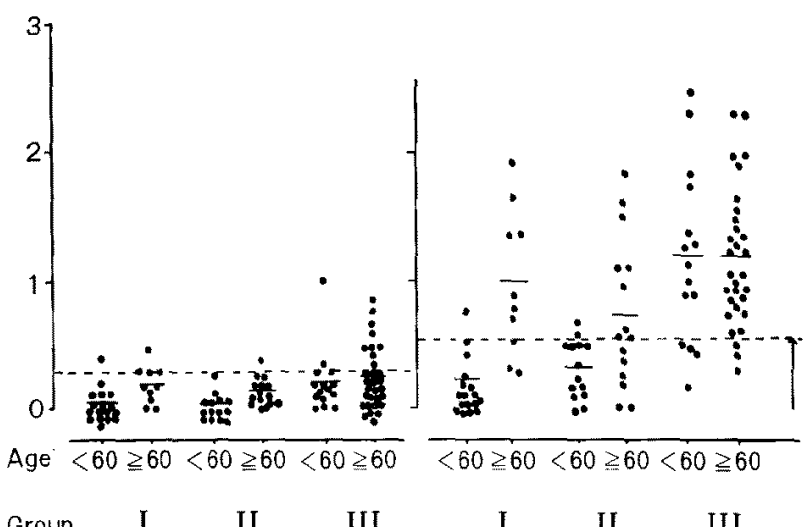

Fig. 2. Renal vascular changes by blood pressure group. 
hyalinization were $0.01 \pm 0.03,0.16 \pm 0.32$, and $0.32 \pm 0.33$ for the younger and $0.03 \pm 0.08,0.04 \pm 0.07$, and $0.31 \pm 0.45$ for the older subjects of Groups I, II, and III, respectively. Hyalinization of small arteries was not substantially different in the age groups for any of the blood pressure groups, and its progression from Group I to III was not as marked as in the arterioles.

(3) Fibroelastic Hyperplasia: In arterioles, the degrees of fibroelastic hyperplasia were $0.06 \pm 0.12,0.04 \pm 0.08$, and $0.21 \pm 0.24$ for the younger and $0.21 \pm 0.20,0.14 \pm 0.10$, and $0.26 \pm 0.24$ for the older subjects of Groups I, II and III, respectively.

In small arteries, fibroelastic hyperplasia was strongly dependent on age. The degrees of fibroelastic hyperplasia were $0.23 \pm 0.16,0.32 \pm 0.22$, and 1.20 \pm 0.68 for the younger and $1.00 \pm 0.56,0.73 \pm 0.46$, and $1.19 \pm 0.53$ for the older subjects of Groups I, II, and III, respectively. The differences in fibroelastic hyperplasia between the age groups were significant in Group I $(\mathrm{p}<0.005)$ and in Group II $(\mathrm{p}<0.05)$. In Group III, fibroelastic hyperplasia was as severe in the younger subjects as in the older, probably because of more severe hypertension in the former than in the latter, as previously mentioned. These findings suggest that fibroelastic hyperplasia of small arteries is more dependent on aging than hypertension.

(4) Renal Vascular Changes and Cardiac Hypertrophy Related to Blood Pressure: The frequency of renal vascular changes and of cardiac hypertrophy in each blood pressure group is illustrated in Fig. 2. Fibroelastic hyperplasia is not included as it was less affected by hypertension. In 18 of 27 cases $(67 \%)$ of Group I, the extent of renal vascular change was within normal limits, with respect to the degree of stenosis and of hyalinization of arterioles and small arteries. The cardiac index was normal in 26 cases (96\%). There were 16 cases $(63 \%)$ in which both renal vascular changes and the cardiac index were within normal limits. In other words, even in those cases with normal blood pressure, $33 \%$ showed some degree of renal vascular change and $37 \%$ some degree of cardiorenal abnormality. Forty-one per cent of the cases in Group II and $7 \%$ of those in Group III showed no demonstrable renal vascular changes. A normal cardiac index was found in $52 \%$ and $27 \%$ of the cases of Groups II and III, respectively. As morphological manifestations of hypertension, therefore, renal vascular changes are more sensitive than is cardiac hypertrophy.

III. Systalic and Diastolic Blood Pressure Related to Cardiac Hypertrophy and Renal Vascular Changes

In order to determine whether systolic or diastolic blood pressure was correlated with the cardiac index and renal vascular changes, an average 
Table III. Coefficients of Correlation of Blood Pressure with Cardiac Index and with Renal Vascular Changes

\begin{tabular}{l|c|c}
\hline \multicolumn{1}{c|}{ Morphological findings } & Systolic blood pressure & Diastolic blood pressure \\
\hline Cardiac index & $0.64^{*}$ & $0.44^{*}$ \\
Degree of stenosis of arterioles & -0.51 & -0.44 \\
Degree of stenosis of small arteries & $-0.56^{* *}$ & $-0.45^{* *}$ \\
Hyalinization of arterioles & 0.49 & 0.53 \\
Fibroelastic hyperplasia of small arteries & 0.47 & 0.36 \\
$* \mathrm{p}<0.01 \quad * * \mathrm{p}<0.05$ & &
\end{tabular}

blood pressure was obtained for each case from all the determinations made prior to one month before death. The correlation coefficients of systolic blood pressure and diastolic blood pressure with the cardiac index, the degrees of stenosis in arterioles and small arteries, hyalinization in arterioles, and fibroelastic hyperplasia in small arteries are shown in Table III. Although each correlation with both systolic and diastolic blood pressure was statistically significant at a p-value of less than 0.01 , the correlation with the systolic blood pressure was highest for the cardiac index $(r=0.64)$, followed by stenosis of small arteries, stenosis of arterioles, arteriolar hyalinization and fibroelastic hyperplasia of small arteries in that order. The correlation with diastolic blood pressure was highest for arteriolar hyalinization $(r=0.53)$, followed by stenosis of small arteries and arterioles, the cardiac index, and fibroelastic hyperplasia of small arteries. The cardiac index was significantly more closely correlated with systolic than with diastolic blood pressure $(p<0.01)$, and the same was true of the degree of stenosis of small arteries $(p<0.05)$.

\section{Discussion}

The present study was concerned with a quantitative analysis of the degenerative changes of renal arterioles and small arteries in relation to age and hypertension.

The degree of stenosis of the arteries was determined by calculating the ratio of the internal diameter to the thickness of the wall." ${ }^{2}$ The ratio was $2.52 \pm 0.21$ (S.D.) for arterioles of subjects less than 60 years of age and without hypertension; this is somewhat larger than the ratios reported by others. ${ }^{5-7)}$ The ratio was $3.91 \pm 0.59$ for small arteries of the same subjects, indicating that the ratio becomes larger as the size of the arteries increases, as previously reported. ${ }^{7}$ However, hypertension was associated with an increase in the degree of stenosis that was nearly equal for both arterioles and small arteries.

With respect to the effect of aging on the degree of stenosis, some authors 
have reported that only stenosis of small arteries is correlated with aging in hypertensive subjects ${ }^{8}$ and others have stated that stenosis of both small arteries and arterioles progresses with advancing age in normotensive subjects. ${ }^{9}$ Our finding was that the degree of stenosis of arterioles was somewhat more advanced in subjects below 60 years of age than in those aged over 60 in all three blood pressure groups. Stenosis of small arteries was somewhat more advanced in the older than in the younger subjects of Groups I and II, but the reverse was true for Group III. The more severe stenosis at younger ages in Group III may have been due to more severe hypertensive disease being present in the younger subjects. In small arterics the narrowing of the lumen could have been caused by fibroelastic hyperplasia, while in arterioles the changes causing narrowing of the lumen may have been counteracted by a loss of elasticity or an atrophy of the vessel wall, as indicated by the finding that the degree of stenosis of arterioles was somewhat less in the older than in the younger subjects of Group I.

Hypertensive changes in arterioles and small arteries of the kidney are characterized by hyalinization of the initima, hypertrophy and degeneration of the media, and hyperplasia of endothelial cells. ${ }^{10), 11)}$ Boyd ${ }^{12}$ ) has stated that hyalinization of arterioles and disruption and thickening of the internal elastic membranes of small arteries are characteristic of benign hypertension, and that arteriolar necrosis and cellular proliferation of the subintima and media are seen in malignant hypertension, in addition to the above-mentioned findings. Hyalinization was divided into four types by Mukai ${ }^{3)}$ : simple hyalinoid, fibrous hyalinoid, fibrous necrotic hyalinoid, and necrotic hyalinoid thickening. However, necrotic hyalinoid thickening was not considered a specific type for the present study, because in many instances it could not be distinguished from fibrous necrotic hyalinoid thickening. Necrotic hyalinoid thickening, synonymous with angionecrosis ("Angionekrose") ${ }^{13}$ ) or fibrinoid necrosis, ${ }^{\text {14) }}$ may not necessarily be characteristic of malignant hypertension. ${ }^{15)}$ In our series also, necrotic hyalinoid thickening was not closely correlated with the level of blood pressure.

The frequency of changes in renal arterioles has been reported as over $90 \%$ in cases with hypertension. $\left.\left.{ }^{10}-11\right), 15\right)-17$ ) The criteria for hypertension in these studies, however, were not standardized. In contrast, changes have been found in over $10 \%$ of cases without hypertension. ${ }^{10), 18)}$ In the present study, a normal range for changes in renal vessels was determined quantitatively, based upon the findings obtained from 17 cases below 60 years of age and without hypertension. An abnormal degree of arteriolar hyalinization then was found in 19\% of cases with normal blood pressure (Group I) and 80\% of cases with persistent hypertension (Group III). If any degree of hyaliniza- 
tion was considered abnormal, the frequency increased to $52 \%$ and $89 \%$, respectively. The differences in the frequencies of renal vascular changes found by different investigators may have been caused by the lack of standardization of criteria for hypertension in their studies, or by differences in the material examined.

In our study, renal vascular changes (stenosis and hyalinization) were within normal limits in $41 \%$ of cases with intermittent hypertension (Group II), and in $7 \%$ of cases with persistent hypertension (Group III). The frequency of no demonstrable renal arteriolar change in hypertension was reported to be $7 \%$ by Castleman \& Smithwick ${ }^{11}$ and $39.8 \%$ by Smith. ${ }^{19)}$ The criteria used for hypertension by Castleman and Smithwick were a systolic pressure of $200 \mathrm{~mm} . \mathrm{Hg}$ or more (average $210 \mathrm{~mm} . \mathrm{Hg}$ ) and a diastolic pressure of $100 \mathrm{~mm} . \mathrm{Hg}$ or more (averagc $130 \mathrm{~mm} . \mathrm{Hg}$ ). Smith used a diastolic blood pressure of $90 \mathrm{~mm} . \mathrm{Hg}$ or more or a heart weight of over $400 \mathrm{Gm}$. (male) or $350 \mathrm{Gm}$. (female) as evidence for hypertension. For the hypertensive subjects of our series (Group III) the mean systolic blood pressure was $178 \mathrm{~mm}$.Hg and the mean diastolic blood pressure was $101 \mathrm{~mm} . \mathrm{Hg}$; both of these are considerably lower than those of Castleman and Smithwick. ${ }^{11}$ Since the frequency of no renal vascular changes in our series was nearly the same as that of Smithwick and Castleman ${ }^{11}$ despite the differences in blood pressure of the subjects, and since it was much lower than that found by Smith, ${ }^{19)}$ the renal arterioles might have been more vulnerable in our subjects than in Americans (if there is not much discrepancy in the interpretation of renal vascular changes).

It has been reported that degenerative changes of small arteries and arterioles in the kidney progress with age even in the absence of hypertension. ${ }^{10), 18)}$ Mukai $^{3)}$ has stated that hyalinization progresses with age even in normotensive subjects, although to a lesser degree than in hypertensive subjects. In hypertensive subjects the degree of hyalinization is severe and progresses more rapidly with age up to 40 years, but its progression with age is not marked in those over 40 years, despite blood pressure levels that are almost the same as those of younger subjects. According to our results, the degree of arteriolar hyalinization was significantly higher in subjects of Group I (normotension) over 60 years of age than in those less than 60 , but the reverse was true for Group III. In Group III severe diastolic hypertension was more frequently found in younger subjects. It can be said from these findings, together with the report of Mukai, ${ }^{3)}$ that a rapid increase in blood pressure is a factor predisposing to arteriolar hyalinization. 


\section{ACKNOWLEDGMENT}

The authors are indebted to Dr. Kenzo Tanaka, Professor of Pathology, Faculty of Medicine, Kyushu University, for his encouragement and criticism. They are also grateful to Dr. Arthur G. Waltz, Section of Neurology, Mayo Clinic, for his reviewing the manuscript.

\section{ReFERENGES}

1. WHO: Arterial hypertension and ischemic heart disease, preventive aspects, report of an expert committee. WHO techn. Rep. Ser. No. 231, 1962.

2. Kernohan, J. W., Anderson, E. W., and Keith, N. M.: Arch. Int. Med. 44: 395, 1929.

3. Mukai, H.: Nagasaki Igk. Z. 35: 140, 1960 (in Japanese).

4. Sato, K.: Hirosaki Med. J. 6: 1, 1955 (in Japanese).

5. Pilcher, J. F. and Schwab, E. H.: Texas State J. Mcd. 28: 665, 1933.

6. Cain, E. F.: Arch. Int. Med. 53: 832, 1934.

7. Tsuji, M.: Acta Gerontol. Jap. 34: 11, 1961 (in Japanese with English abstract).

8. Masaki, T.: Jap. J. Nephrol. 3: 211, 1961 (in Japanese with English abstract).

9. Ikeda, M., Fujii, J., Kuramoto, K., Murata, K., Kaneko, J., Ozawa, T., Terasawa, F., Hosoda, S., Kurihara, H., Kimata, S., Lie Hon Ying, and Matsushita, S.: Acta Gerontol. Jap. 38: 27, 1963 (in Japanese with English abstract).

10. Moritz, A. R. and Oldt, M. R.: Am. J. Path. 13: 679, 1937.

11. Gastleman, B. and Smithwick, R. H.: J.A.M.A. 121: 1256, 1943; New Engl. J. Med. 239: $729,1948$.

12. Boyd W.: Textbook of Pathology, 7th Ed., Lea \& Febiger, Philadelphia, p. 517 \& 594, 1961.

13. Matsuoka, S.: Tr. Soc. Path. Jap. 28: 381,1938 (in Japanese).

14. Pickering, G. W.: High Blood Pressure, Grune \& Stratton, New York, p. 235, 1955.

15. Sommers, S. C., Relman, A. S., and Smithwick, P. H.: Am. J. Path. 34: 685, 1958.

16. Fishberg, A. M.: Arch. Int. Med. 35: 650, 1925.

17. Bell, E. T. and Clawson, B. J.: Arch. Path. 5: 939, 1928.

18. Bell, E. T.: Renal Diseases, 2nd Ed., Lea \& Febiger, Philadelphia, p. 337, 1950.

19. Smith, J. P.: J. Path. Bact. 69: 147, 1955. 